\title{
Assessment and Characterizing Mechanical Damage in Packaged Bananas in the Post-harvest Supply Chain
}

\author{
Fernando I., 2, a, Fei J. 1,2, Stanley R. ${ }^{2,3}$ and Enshaei $\mathrm{H}^{1,2}$ \\ ${ }^{1}$ National Centre for Ports and Shipping, \\ Australian Maritime College, University of Tasmania, Australia. \\ ${ }^{2}$ ARC Training Centre for Innovative Horticultural Products, University of Tasmania, Australia. \\ ${ }^{3}$ Centre for Food Innovation, Tasmanian Institute of Agriculture (TIA), University of Tasmania, Australia
}

\begin{abstract}
Quality deterioration in packaged bananas caused by mechanical damage along the post-harvest supply chains (SC) remains obscure until the packages are unpacked for sale at the retail stores and therefore, the mechanism of damage occurrence remains unclear. This study assessed the mechanical damage levels of bananas in 300 cartons from pack houses to retail stores in Australia. The damage across the SC were shown to be progressive across the SC. Bruising and neck damage levels in bananas increased from the distribution centre (DC) to the retail stores. Mechanical damage in unripe bananas was influenced by the package location in the stacked-pallet, and the transport and handling of packages within the last-mile of the SC (DC to retail) further exacerbated the damage. This study further characterized the damage development by subjecting packaged bananas to simulated vibration, top-load compression and drop impact. It was revealed that the exposure to vibration resulted in rubbing; top-load package compression contributed to bruising and, the drop impact caused severe neck injuries in bananas. The knowledge of the damage incidences across the SC and causes of damage may contribute to the development of interventions targeted at improving the quality of bananas in the post-harvest SC in Australia.
\end{abstract}

\section{Introduction}

In most post-harvest supply chains (SC), a certain degree of mechanical damage to fresh produce during the distribution is unavoidable. Appearance quality of fruits is a major determinant for the consumer's willingness to purchase [1]. Previous studies have shown that cosmetic damages in bananas resulted in poor consumer acceptance and marketability [2]. Bananas have been crowned as the most sold supermarket produce in Australia [3] and their quality is of a great interest not only to the banana industry but also to the major supermarket chains which account for over $74 \%$ of the total banana sales [4] in Australia. Bananas, like any other fruit are subjected to mechanical stresses from harvest through to consumers which is one of the major factors for the post-harvest quality deterioration [5-7]. It was estimated that $5-8 \%$ of the gross volume of bananas has been accounted as wastage in the post-harvest SC in Australia which amounts to AU\$ 46-73 million per annum [8]. Therefore, mechanical damages in bananas have been a significant concern for both the banana and retail industries in Australia, requiring a systematic investigation.

Like other fresh produce, bananas also exhibit a degree of physio-chemical changes due to mechanical injury. One of the most significant and immediate

\footnotetext{
a Corresponding author: Indika.fernando@utas.edu.au
}

consequence of mechanical damage is the attack of microorganisms, which may cause further decay in fruits [9]. Mechanical damages cause browning in bananas due to the oxidation of poly phenols [10]. Maia, et al. [9] found that mechanical damage caused higher enzyme activity in the affected area followed by alternations in colour, flavour, rapid tissue softening and induced ripening. It was also showed that mechanical damage induced respiration levels in bananas $[7,10]$. Chukwu, et al. [11] further reported that abrasion damage caused rapid moisture and weight loss compared to impact bruising in bananas. Similarly Maia, et al. [10] reported that abrasion and cut damages resulted in the highest fresh weight loss in bananas compared to the other treatments. Furthermore, Maia, et al. [10] showed that mechanical damage expedited ripening and influenced the rate of conversion of starch into total soluble sugars (TSS) in the pulp. These findings conclude that mechanical injury induce ripening, hence reduce the effective shelf-life of the fruit. The most significant consequence of these alterations would be the implications on fruit quality and the perceived value of bananas, which can directly affect the marketability.

Mechanical damage in fruits can occur due to compression, impact and vibration when fruits are transported in packages or in bulk bins [12-14]. Bruising can occur due to impacts or compression forces $[15,16]$ resulting in peel and/or flesh damage in bananas 
depending on the severity of the impact/pressure. Damage caused by friction occurs due to the relative movement of the fruit against other contacting surfaces. Abrasion damages such as rubbing in bananas can occur due to vibration or when bananas are handled in clusters (e.g. when clusters are rubbing against conveyer belts in a packing line). Several studies showed that vibration during transport was a critical factor for the development of mechanical damage in bananas $[6,17,18]$ and prolonged exposure to vibration could escalate the incidence of damage [5]. Studies on other packaged fruits revealed that the vibration damage can be severe in loosely packed fruits compared to the tightly packed fruits $[19,20]$. Unlike most other fruits, bananas may also exhibit stem end tearing or neck damage. Neck injuries are caused by separation of stem from the fruit [21] which can affect the quality and the consumer acceptance at the retail stores [2]. However, limited knowledge exists on the exact mechanism of the occurrence of neck injuries especially when banana clusters are packed inside corrugated paperboard cartons during distribution.

Studies assessing the occurrence of mechanical damage in bananas across the $\mathrm{SC}$, from the farm gate to the retail-shelf are limited. Maia, et al. [22] investigated mechanical damage at four distinct locations in the banana SC (pre-selection, after packing, after transport and retail market) and concluded that the damaged levels increased and accumulated along the chain. A similar study revealed that the damage to bananas was progressive along the $\mathrm{SC}$ and the highest damage occurred during the post-harvest distribution [23]. However, damages caused by different mechanical stresses in bananas are not well defined in literature and a deep understanding of different types of damages is necessary to determine the causes of such damage. Previous studies revealed several types of cosmetic damages in bananas (Fig.1) [2] however their mechanism of occurrence, especially within a package and during the handling across SC has not been well understood. Therefore, this study aims to (1) assess and quantify the mechanical damages in bananas across the post-harvest SC in Australia and (2) characterize different types of damages in packaged bananas caused by induced mechanical stresses including vibration, compression and transient impacts.

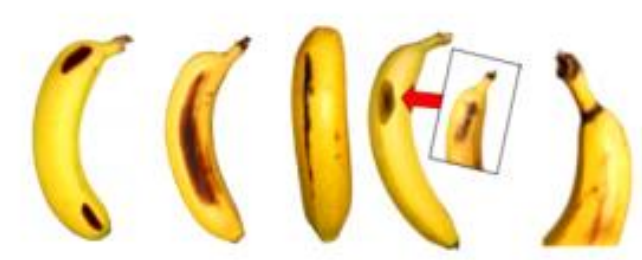

Fig 1: Different Types of Cosmetic Damages in Bananas [2]

\section{Materials and Methods}

\subsection{Damage Assessment in Packaged Bananas}

To understand the occurrence of cosmetic damages in bananas, the damage levels were assessed in three locations across the chain, from the pack-houses through the distribution centre (DC) to the retail stores (Fig.2). Bananas are commonly packed in three grades (i.e. premium, extra-large and large) for different markets and the 'premium' grade (selected and packed as the highest grade) is intended for the major supermarket chains in Australia. Cartons packed with 'premium' grade bananas were selected for this study because they are generally graded before the packing process with minimal preexisting damage. Having a minimal (or none) existing damage in bananas at the initial stage of the SC (i.e. packhouse) is essential for the damage comparison to distinguish the damage development at the later stages of the $\mathrm{SC}$ (i.e. DC and retail stores).

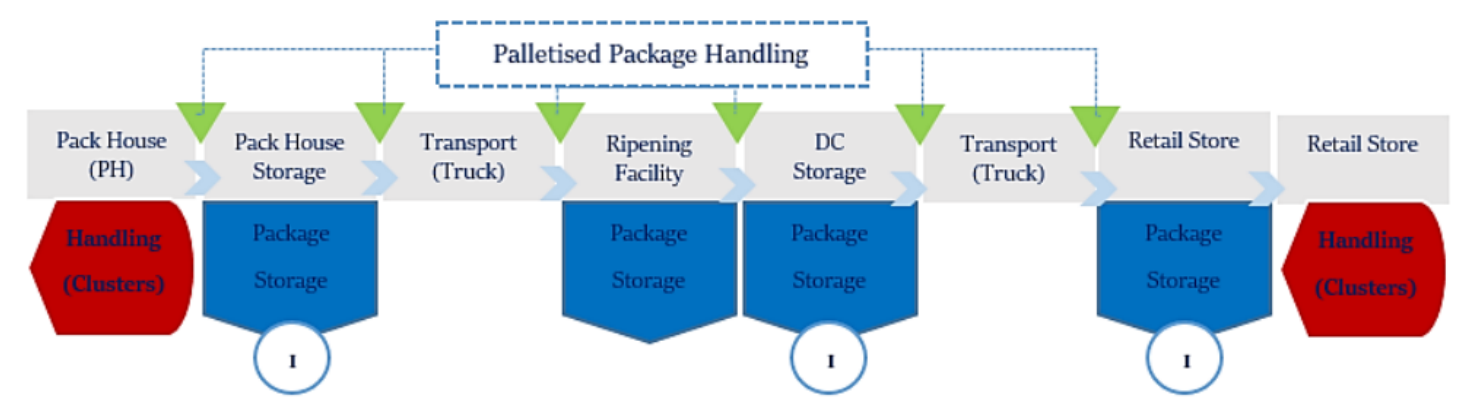

Figure 2: Simplified Post-Harvest Banana Supply Chain [(I) - Damage inspection points]

Five SCs were selected for the study, originating from five pack houses in Northern Queensland to the retail stores in Melbourne (over $3000 \mathrm{~km}$ via the road route) through a regional DC in Melbourne. A sample of 100 packages were selected randomly in each location (i.e. pack-house, DC, retail store) for damage assessment. Twenty packages were assessed in each pack house and retail store. The stacked-tier of the selected packages in the pallets in each location is given in Table 1. Banana packages were stacked in 10 tiers on wooden pallets 
during the distribution from the pack-houses to DC and stacked only up to five tiers (mostly consolidated with other produce packages) from the DC to retail stores. Therefore, number of cartons inspected in each tier was 10 and 20 in the DC and retail stores respectively. A total of 300 cartons packed with 3,240 clusters of bananas $(15,261$ individual fingers) were assessed for mechanical damage and quality defects across the SC.

\subsection{Characterizing Mechanical Damage in Packaged Bananas}

Packaged bananas were subjected to simulated vibration, top-load compression and simulated handling (drop impact) to determine the causes of different types of mechanical damage in bananas. Forty (40) sample banana cartons were arranged for the experiments. All sample cartons were sourced from the same batch of fruits, harvested/packed from the same farm/pack-house in Tully, Queensland. Fifteen (15) cartons were packed with unripe bananas (stage two) and the rest were packed with ripe bananas (stage six) as per the ripeness chart in Ekman, et al. [2]. The cartons were packed in three-layers (5-7 clusters per layer arrangement) inside each package. All sample cartons were pre-inspected for existing damage and the clusters with pre-existing damages were replaced with non-damaged clusters prior to each experiment. All cartons with unripe bananas were conditioned for 48 hours at $13.5{ }^{\circ} \mathrm{C}$ and $50-60 \%$ relative humidity (RH) and the ripe bananas were stored in 15-18 ${ }^{\circ} \mathrm{C}$ at $90-100 \% \mathrm{RH}$ (inside the ripening chamber) for 48 hours before the experiments.

\subsubsection{Simulation of Package Handling and Top-load Compression}

A drop-testing machine (Lansmont PDT 80, CA, USA) was used to drop packaged bananas (ripe) from heights of $0.3 \mathrm{~m}$ and $0.5 \mathrm{~m}$ (Fig. 3). Each carton was dropped from these specified heights three times during each test and the experiment was conducted in a randomized block design in pentaplicates $(5 \times 2)$. For the package compression experiment, ten cartons of ripe bananas were subjected to top-load compression by a compression testing machine (Instron 1185, MA, USA). Five sample cartons with ripe bananas were crushed to $16 \mathrm{~mm}$ and the other five cartons were crushed to $25 \mathrm{~mm}$ vertical displacement levels. Each package compression experiment was conducted in randomized block design in pentaplicates. All the sample packages were stored in $13.5^{\circ} \mathrm{C}$ and $50-60 \% \mathrm{RH}$ and the damage levels in packaged bananas were assessed 12 hours after each experiment by the procedure described in section 2.3 to derive a damage score for each package.
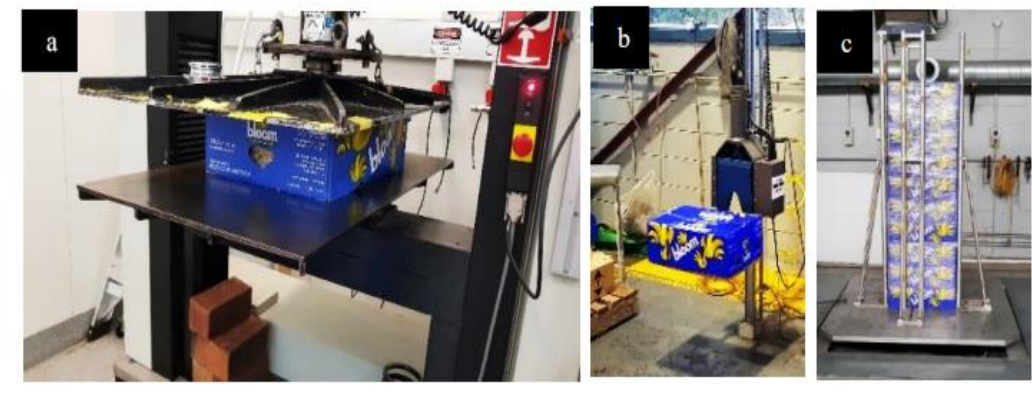

Figure 3: Characterizing Mechanical Damage by (a) Top-load Compression, (b) Drop Impact and (c) Simulated Vibration

\subsubsection{Simulated Vibration Testing}

A single vertical column of ten banana cartons (unripe) was subjected to simulated vibration intensity of $0.36 \mathrm{~g}$ for a duration of 3 hours by an electro-hydraulic vibration simulation table. The vibration profile of ASTM-D4169 was used[24]. The column was triple-stretch-wrapped with transparent stretch film and supported by vertical steel columns fixtures, attached to the table from either side to avoid the collapsing of the packages during the test (Fig.3). After each experiment the cartons in the bottom $\left(1^{\text {st }}\right.$ tier $)$, middle $\left(5^{\text {th }}\right.$ tier $)$ and top $\left(10^{\text {th }}\right.$ tier $)$ were removed from the column for damage assessment and the three sample positions were stacked with fresh (nonvibrated) cartons. The packages in the rest of the positions of the column were reused as dummy packages 
in each repeat experiment. The damage level in bananas stacked in the three sample positions of the column were evaluated 12 hours after each experiment by the procedure described in the section 2.3. This experimental procedure was repeated for ripe bananas. All vibration simulation experiments were conducted in pentaplicates in a randomized block design $(3 \times 5)$.

\subsection{Damage Assessment and Analysis}

The damage in packaged bananas was assessed by a 0-4 point hedonic scale (0- No damage; 1 - Trace damage; 2- Slight Damage; 3- Moderate Damage; 4- Severe Damage) in accordance with the visual quality assessment chart [2]. A visual damage index (VDI) score was calculated by the Eq.1 for each package similar to

\section{Results}

\subsection{Mechanical Damage along the Supply Chain}

Different types of mechanical damages exhibited in different stages of the SC during the damage inspection is given in Fig. 4. The levels of neck damage, bruising and various abrasion damages (i.e. scuffing, fruit rub, and blackened rub) by the stacked-tier in the pallet as revealed in the DC and retail stores are given in Figure 5 and Figure 6 respectively. Damage assessment in packaged bananas revealed that the accumulated damage level was escalated by $1.8 \%$ to $8.9 \%$ from the pack houses to the the equivalent bruise index for apples [25]. The mean VDI score was used for the comparison of damage levels in bananas occurred after each treatment.

VDI $(\%)=(0.1 \times$ Trace Damages $(\%)+0.2 \times$

Slight Damages $(\%)+0.7 \times$ Moderate Damages

$(\%)+1.0 \times$ Severe Damages (\%) (Eq.1)

One-way analysis of variance (ANOVA) and Turkey's Honest Significant Difference (HSD) test were performed to test for significant differences within the means by GraphPad® Prism 7 statistical analysis package (GraphPad Software, La Jolla, CA, USA).

retail stores (Fig. 4). The highest increment in damage $(5.1 \%)$ was revealed in abrasion type damage while bruising and neck damage exhibited a relatively less increment $(<2 \%)$ from the pack houses to the retail stores. The highest increment of damage between two SC nodes was revealed in abrasion damage between the pack houses and DC (2.6\%) and the abrasion damage level has further worsened between the DC and retail stores $(2.5 \%)$. The level of bruise and neck damage at the pack houses marginally increased at the DC however neck damage levels had nearly doubled between the DC and retail stores.

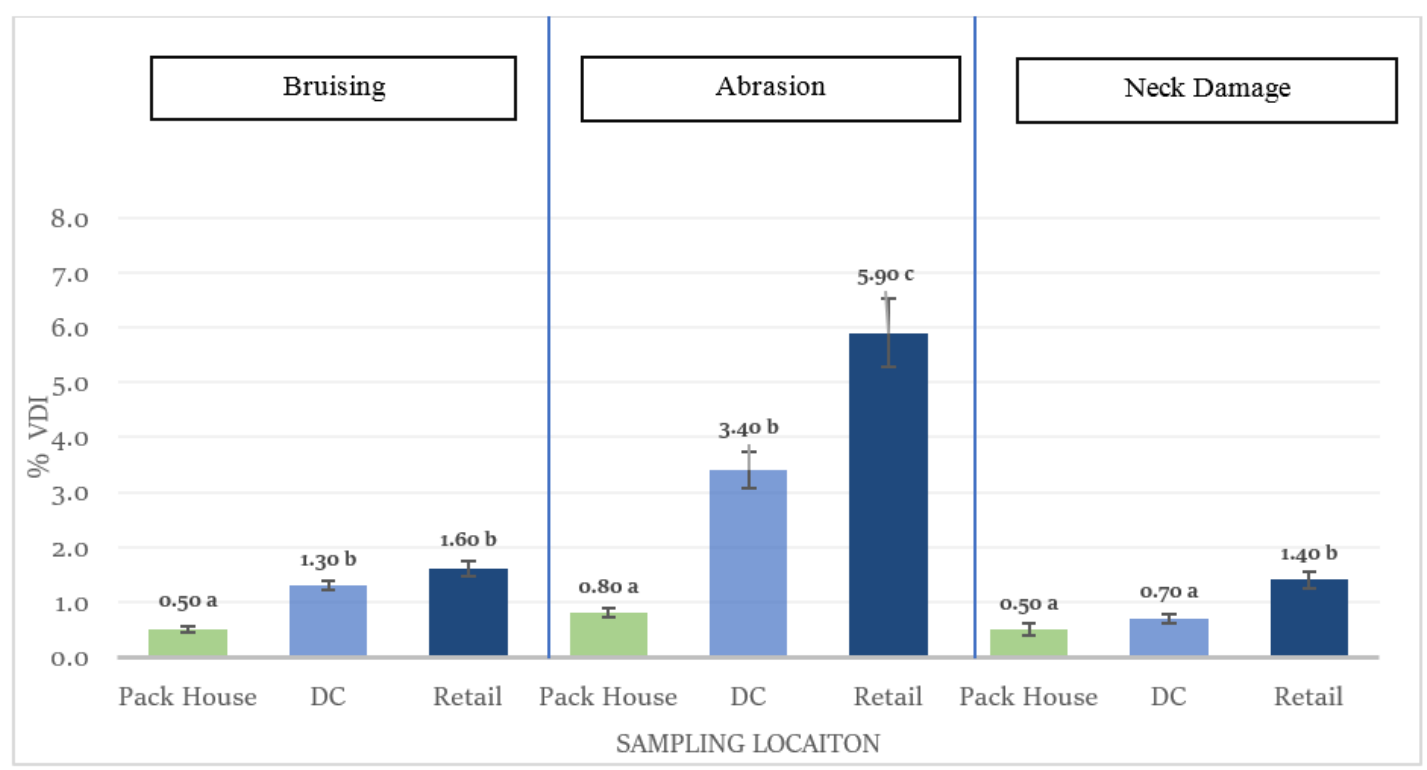

Figure 4: Mechanical Damages in Bananas by the Sampling Location in the Supply Chain [For each damage Category Different
Characters Indicates Significantly $(\mathrm{P}<0.01)$ Different Results]

Figure 5 illustrates the different types of damage in bananas by the stacked-tier which were revealed during the damage assessment at the DC. The best-fitted line $\left(3^{\text {rd }}\right.$ order polynomial) for the overall damage level from tier one to ten elaborates that the highest damage level in a stacked pallet was found in the most top and the bottom packages. The accumulated damage level is minimal in the mid-tier packages of the pallet. Abrasion damage largely contributed to the escalation of the overall damage level in the two bottom tiers and the three top tiers. It is also revealed that the bruise damage and neck injury levels decreased from the bottom to top of the pallet. Statistical analysis revealed that the bruise damage levels in the first six tiers are significantly different $(\mathrm{P}<0.01)$ to that of the top four tiers. Similarly, the neck damage levels of the top four tiers are significantly different 
$(\mathrm{P}<0.01)$ to that of the rest of the lower tiers in the pallet. The abrasion damage level in the most bottom tier is significantly different $(\mathrm{P}<0.01)$ to the rest of the tiers except for the damage level in the ninth tier. There was no statistical difference in abrasion damage levels from the third tier to the seventh tier however, the highest abrasion damage level was revealed in the top tier packages which was statistically different $(\mathrm{P}<0.01)$ to the rest of the tiers. Most of the abrasion damage levels were influenced by the frequent occurrence of fruit rubbing and scuffing especially in the most bottom and top tiers of the pallet.

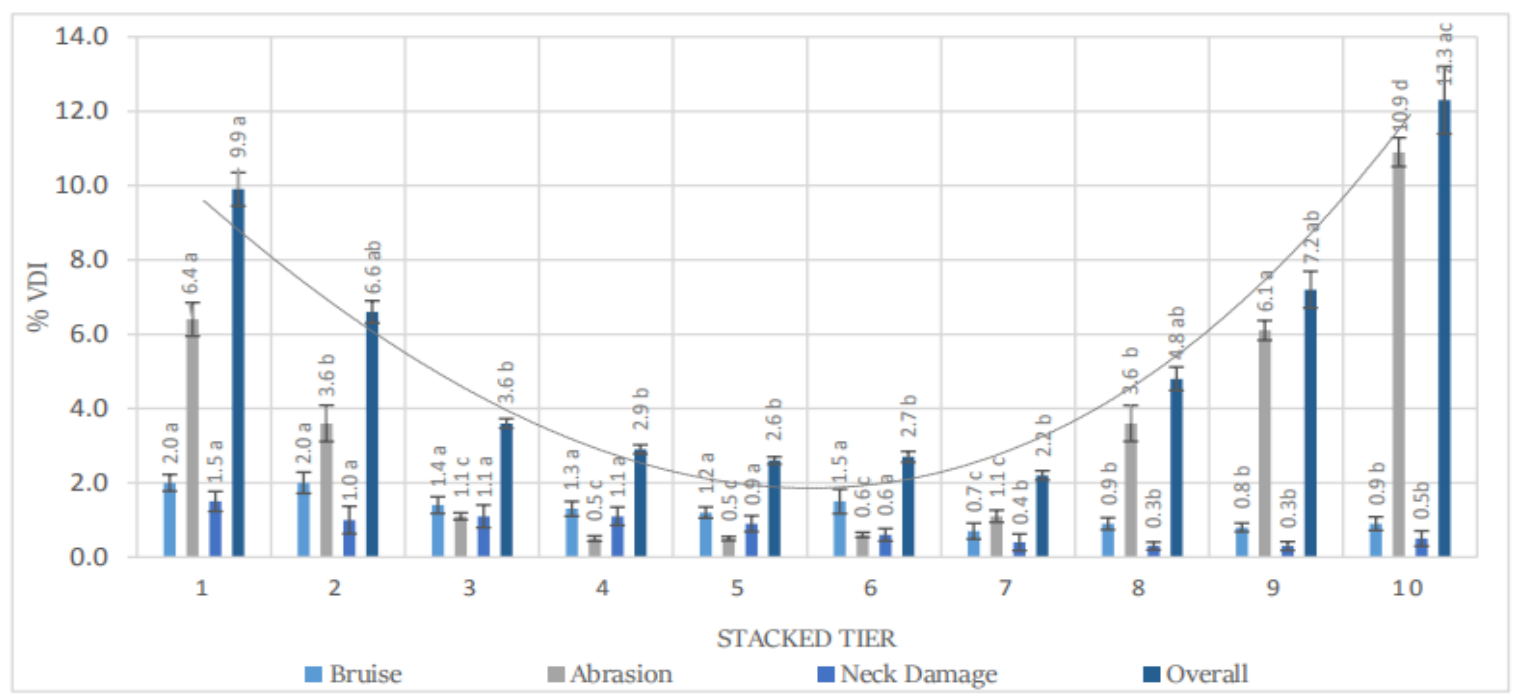

Figure 5: Mechanical Damages in Bananas by the Stacked Tier at the DC [For each damage Category Different Characters Indicates Significantly $(\mathrm{P}<0.01)$ Different Results]

As exhibited in Fig. 6 there are no statistically significant differences found for any damage type or for the overall damage level based on the stacked tier of the packages at the retail stores. It was also revealed that, at the retail stores the most critical damage was abrasion. As illustrated in Fig. 7, the most frequent abrasion damage at the retail stores was rubbing damage $(70 \%)$ including the fruit rub and blacked rub however, the other abrasion damages such as scuffing (17\%) and scars (13\%) were marginally exhibited.
Inside each package, bananas are packed in three layers (Fig. 8) and the damage level by the layer of fruit inside the carton is given in Fig. 9. It was revealed that a higher incidence of bruising (1.2\%) existed in the first layer of fruits inside the carton and the frequency of abrasion type damages such as fruit rub and scuffing was higher in the second and third layer inside the carton. Neck injuries were also escalated in the third layer and the second layer $(1.3 \%)$ than the first layer $(0.1 \%)$.

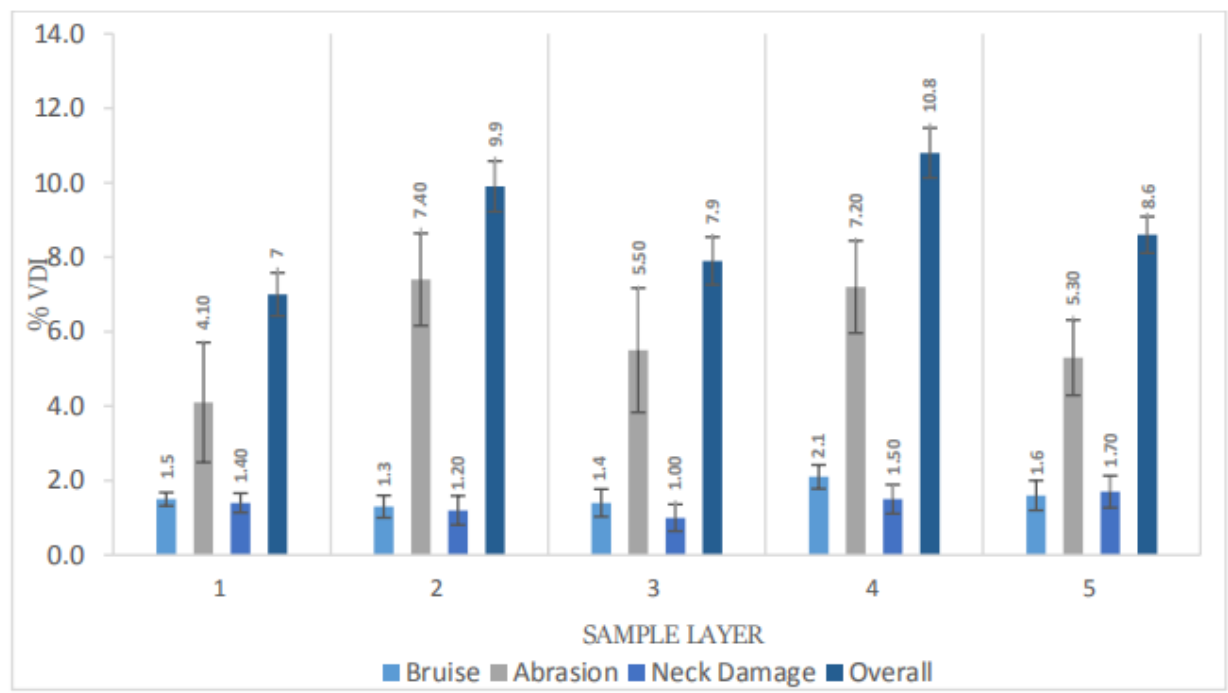

Figure 6: Overall Mechanical Damages in Bananas recorded during Random Inspection at each Stage of SC [No statistically different results $(\mathrm{P}<0.01)$ for each damage category have been exhibited] 


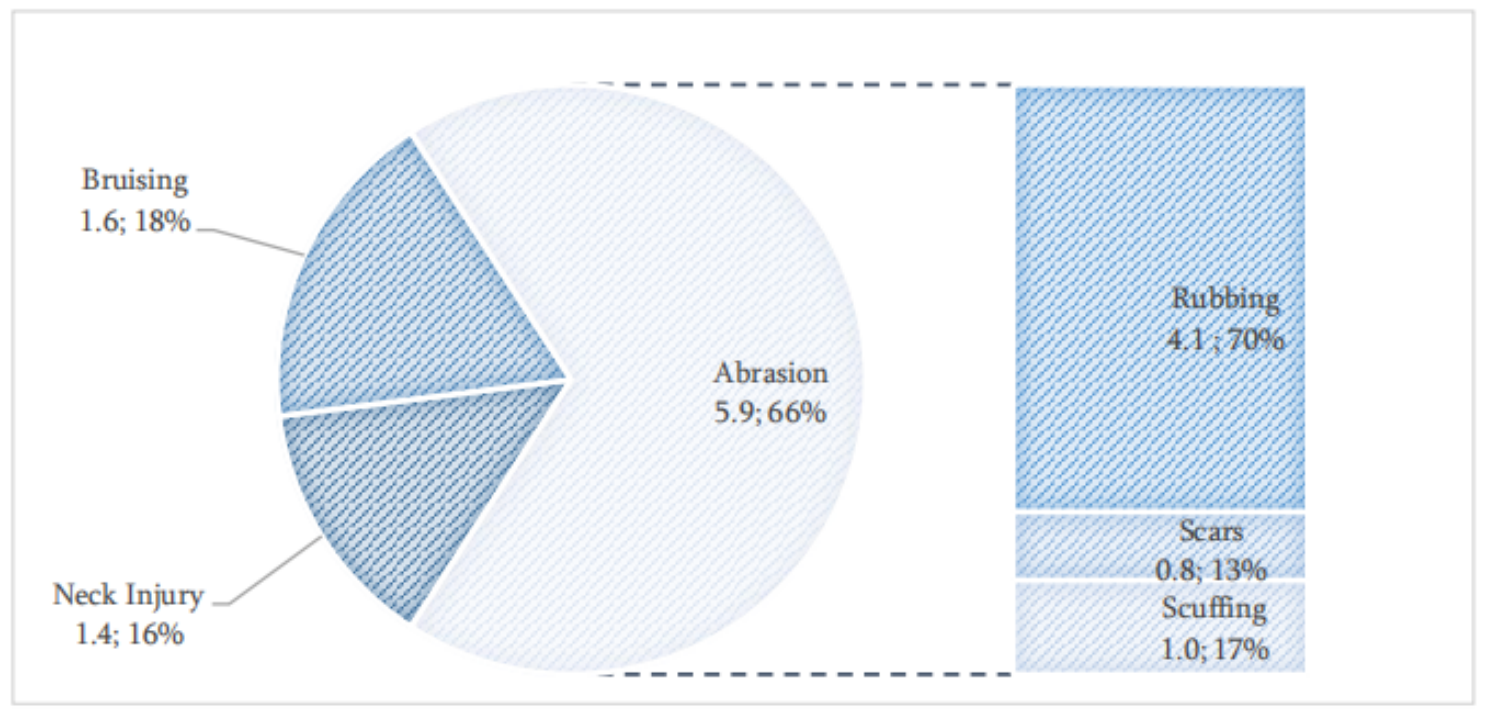

Figure 7: Breakdown of VDI Score and the Percentage (\%) by Each Damage Type for the Retail Stores

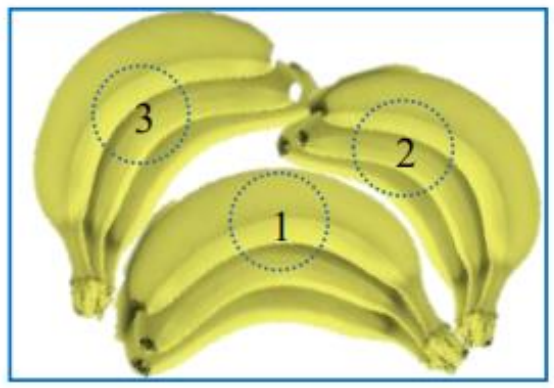

Figure 8: Spatial arrangement of bananas inside the corrugated box packed in three layers (Layer 1, 2 and 3)

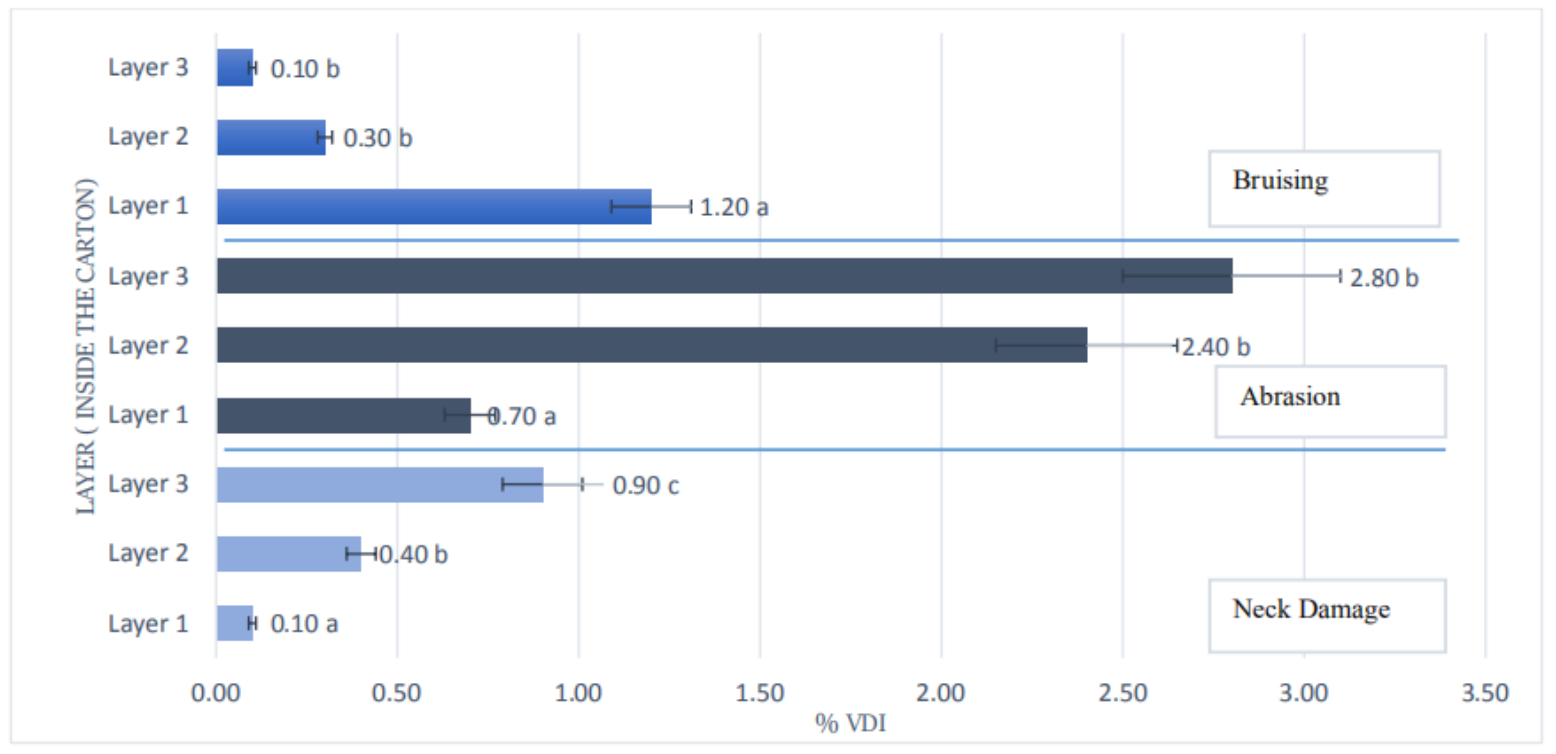

Figure 9: VDI for the Layer of Bananas inside the Carton (Retail) [Different characters (a, b and c) denotes statistically different $(\mathrm{P}<0.01)$ results for each damage category] 


\subsection{Characterizing Mechanical Damage in Packaged Bananas}

Different types of mechanical damages occurred in packaged bananas due to package drop, top-load compression and vibration (Fig. 10). Package drop mainly resulted in neck injuries and package compression resulted in bruise damage (Fig. 10). In addition to neck injuries, package drop occasionally resulted in minor levels of bruising in ripe bananas. Exposure of packages to simulated vibration mainly resulted in abrasion damage in bananas (Fig. 11). For the same vibration treatment, the damage levels were significantly higher $(\mathrm{P}<0.05)$ in ripe bananas compared to the unripe (green) bananas (Fig. 11) and majority of the vibration damage occurred in the top two layers of bananas inside the package.

As illustrated in Fig. 12, the neck damage level (VDI \%) worsened $(\mathrm{P}=0.076)$ with the increasing drop height of the packages. Nearly $94 \%$ and $91 \%$ of the neck injuries (attributed to $0.3 \mathrm{~m}$ and $0.5 \mathrm{~m}$ drop heights respectively) were concentrated in the top two layers of bananas inside the package (Fig.12). Bruise damage level (VDI \%) in bananas significantly $(\mathrm{P}<0.05)$ proliferated due to the increased top-load compression of the packages from $16 \mathrm{~mm} 25 \mathrm{~mm}$ (Fig. 12). Nearly $72 \%$ of bruising damage occurred under the $16 \mathrm{~mm}$ compression and $84 \%$ under the $25 \mathrm{~mm}$ compression was localized in the first layer inside the package.

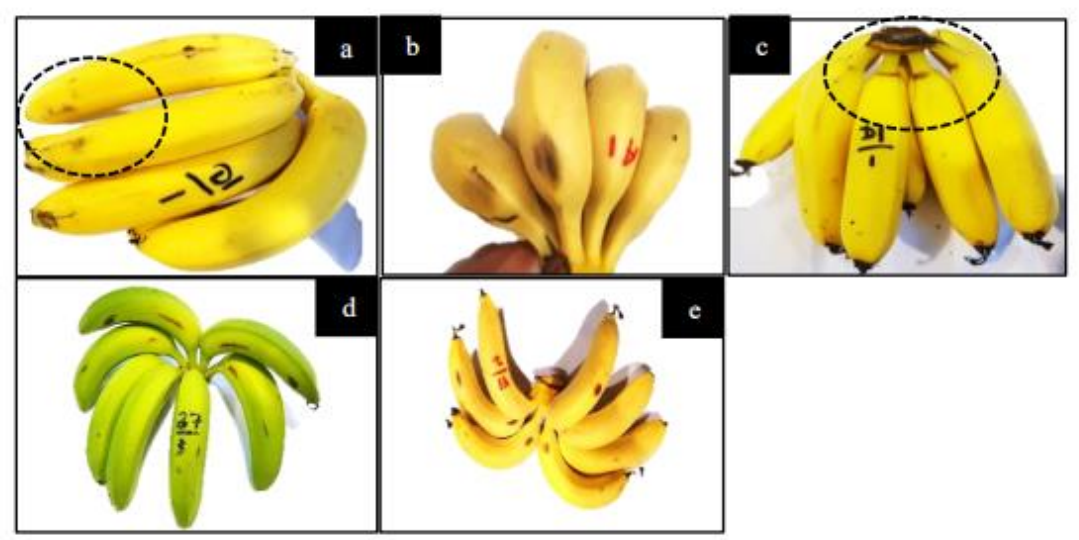

Figure 10: Different types mechanical damages occurred in bananas:

(a) minor (trace) levels of bruising occurred by the package drop impact (b) shoulder bruising in bananas occurred by top-load compression $(25 \mathrm{~mm})(\mathrm{c})$ severe neck damage occurred due to package drop impact (d) fruit-rub damage in unripe (green) bananas caused by simulated vibration and

(e) fruit-rub damage in ripe bananas

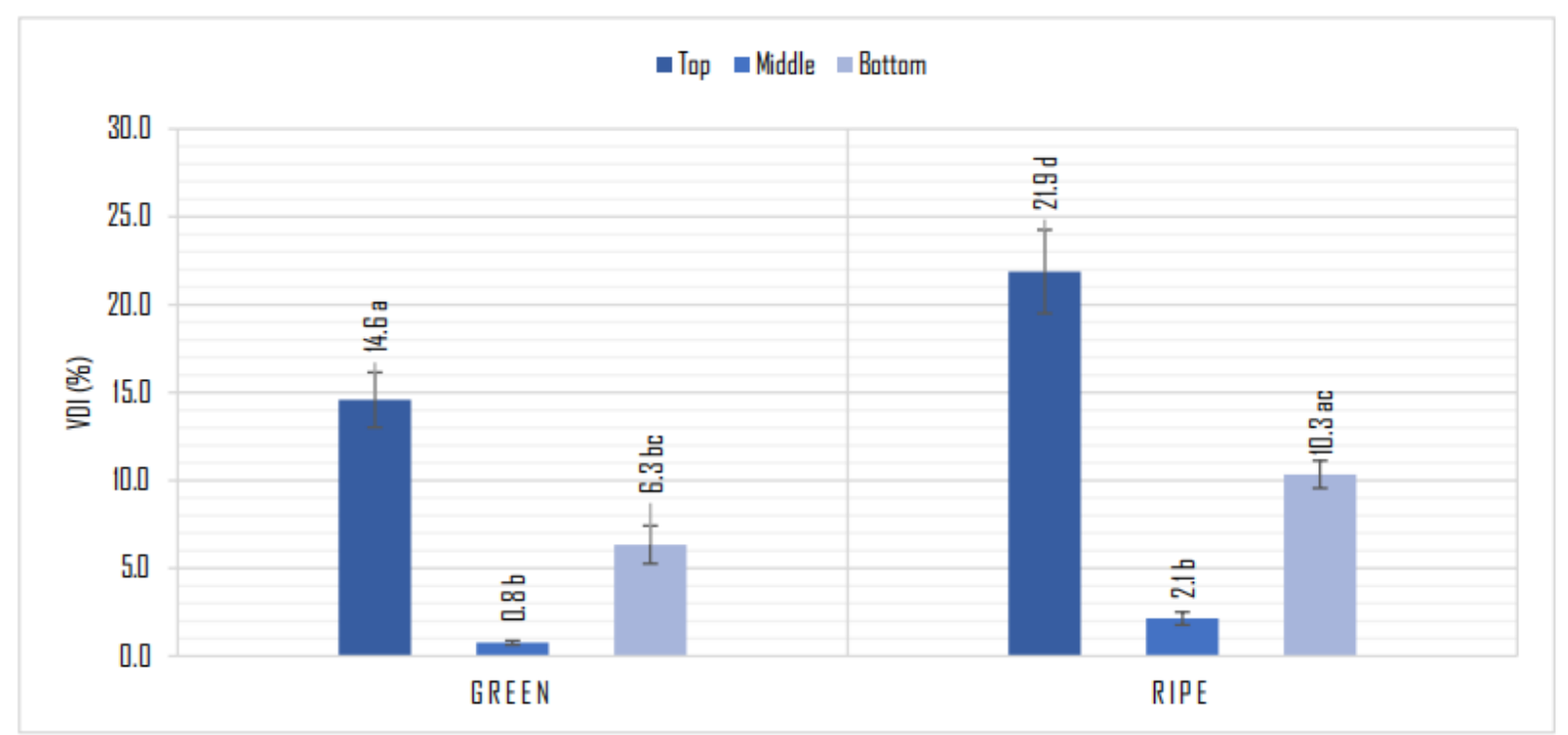

Figure 11: Mechanical damage levels (i.e. abrasion) in green and ripe bananas caused by simulated vibration [Different letters indicate significantly different results $(\mathrm{P}<0.05)$ ] 


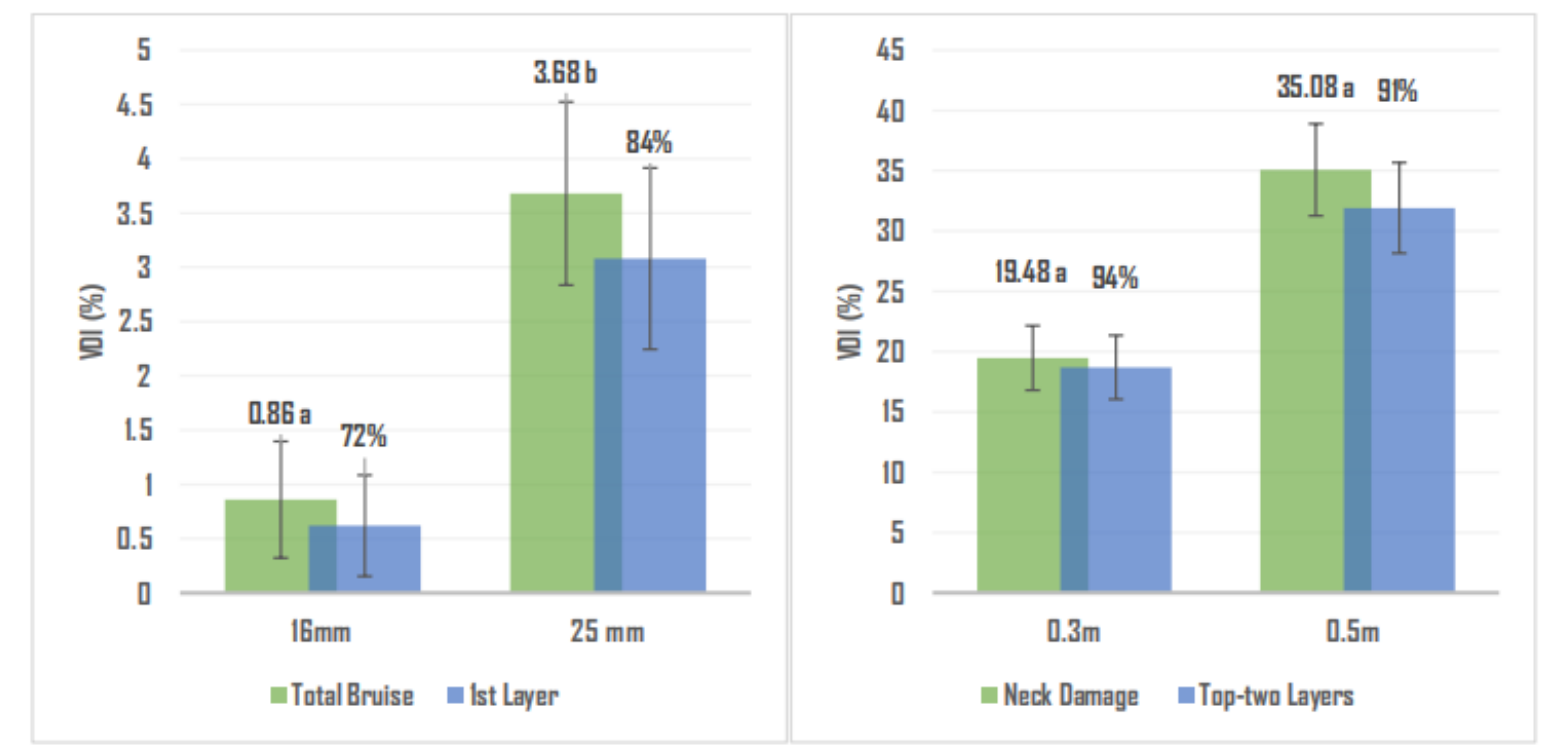

Figure 12: Mechanical damage levels occurred during the top-load compression (left) and drop-impact tests (right) [Different letters indicate significantly different results $(\mathrm{P}<0.05)$ in each test]

\section{Discussion}

The results from the damage assessment indicates that all types of damages in bananas (i.e. bruising, neck injuries, abrasion) exacerbated along the SC, resulting in poor visual quality at the retail stores. At all three stages of the SC, abrasion damages were the most frequently reported. It was also revealed that most fruit rub damages occurred between pack houses and DC while blackened rub and scuffing damages were mostly caused from DC to retail stores. These results indicate that damages in bananas are accumulating along the SC, leading to an overall quality deterioration at the retail end. In addition, abrasion damage is the predominant type of damage in post-harvest banana SC. Similar results were reported in previous studies which reported that mechanical damages in bananas were progressive along the $\mathrm{SC}[9,10,22,23]$ and the abrasion type damages were predominant in packaged bananas [2].

The simulation tests revealed that ripe bananas were more susceptible to vibration damage compared to the unripe (green) bananas (Fig. 11). This could be the reason for the disproportionate increment of damage during the last-mile distribution of ripe bananas (from DC to retail). The results show that the interstate transport and handling of bananas (over several thousand kilometers) resulted in $2.6 \%$ damage increment in unripe (green) bananas while the distribution of ripe bananas to retail stores located less than $100 \mathrm{~km}$ from the DC caused $2.5 \%$ increment in abrasion damage. Similarly neck injuries in bananas nearly doubled between the DC to retail stores.

The drop tests confirmed that one of the major reasons for neck injuries in bananas is the manual handling of packages. The significant increase in neck injuries in the latter part of the SC (from DC to retail) indicates that softer ripe bananas become more susceptible to neck damage than the harder unripe (green) bananas during handling. Therefore, it is essential that packages with ripe bananas are handled cautiously, especially during the palletizing and package consolidation i.e. staking different produce packages together in mix-pallets at the DC to be dispatched to different retail stores to minimize neck injuries. Similar results were reported in the previous studies inciting that the susceptibility of bruising in bananas escalated with ripening $[10,26]$ and thus, carefully handling and storage packages will be integral to improving the quality.

Most of the abrasion damage recorded at the DC was concentrated in the top and bottom tiers of the pallet (Fig.5), while the middle tier packages (tier 4 to tier 7) exhibited a minimal level of abrasion (i.e. fruit rub). Most of the fruit rub damage in the bottom and top tiers of the pallet were caused by relative motion of fruits in the package, which was associated with the transmissibility of vibration in a pallet. The simulated vibration tests revealed similar results, confirming that more vibration damage occurred in the bottom and top tier packages with minimal damage occurring in the middle tiers (Fig. 11). This result coincides with the previous studies which have shown that the highest vibration damage in fruits were often revealed in the most top tier packages of a pallet lcolumn [27, 28]. However, the reason for reduced damage in the middle tiers of the pallet requires further investigation, including examining the vibration dampening properties of corrugated packaging.

Bruising and neck damage levels escalated in the bottom tiers of the pallet, revealing the highest levels of damage in the most bottom tier packages (Fig.5). Topload compression tests confirmed that bruising in bananas occurred mostly due to the compression of the packages from the upper tiers. The weight from the upper tiers act on bananas as quasi-static compression during storage, handling and transport. Dynamic compression forces on packages during transport may also occur due to shocks encountered by palletized packages. However, more research is necessary to determine the influence of 
transient shocks during transport on the occurrence of damage in packaged bananas. Despite the relatively lower bruise and neck damage levels in the upper tiers of the pallet, the overall accumulated damage levels still increased in the top tiers, due to the high levels of abrasion damage (Fig.5).

The presence of increased neck injuries in the bottom tier packages at the DC indicates that neck injuries are not only caused by package handling but also associated with the weight of the fruits stacked on-top of a given package. It was revealed during the damage assessment that occasional package failure in the bottom tiers was closely associated with a higher incidence of neck injuries in bananas. However, the reasons for such sporadic package failure was not always straightforward but observed to be associated with misaligned stacking of packages on pallets and moisture absorption by paperboard cartons during the ripening process.

Most abrasion damage was concentrated in the top two layers inside the package (Fig.9). This can be due to the increased freedom of movement of fruits when exposed to vibration and the movement or bouncing of clusters inside the package due to the shock impacts (e.g. package dropping). When individual fingers are free to move inside the package, it results in rubbing between the fingers and clusters. The weight of the two upper layers and the inter-locking mechanism of clusters inside the package (Fig.8) restricted the freedom of movement for clusters in the first layer of the carton resulting in reduced abrasion damage in the first layer. During the package drop tests, the energy of the transient shock events (e.g. by hitting the floor/ground) was partially transmitted to banana clusters packed inside. The top two layers of fruit (especially the most top layer) had more freedom to bounce resulting high incidence of neck injuries in the top two layers inside the package. Similar results were reported by Ekman, et al. [2].

\section{Conclusion}

Minimizing quality deterioration in bananas requires knowledge on the incidence of mechanical damage along the SC and the mechanism of damage occurrence in packaged bananas. This study evaluated the cosmetic damage levels in bananas along the post-harvest SC and experimentally tested and confirmed the causes for damage occurrences. The damage levels were found progressive along the SC from the pack houses to the retail stores. The damage levels in green bananas were significantly influenced by the package position in the stacked-pallet. The abrasion damage was mostly concentrated in the top and bottom tier packages while both neck injuries and bruise damage were frequently found in the middle and lower tier packages of the pallet. All types of damages further proliferated during the distribution of ripe bananas to the retail stores and bruising and neck injury levels nearly doubled from DC to retail stores. The laboratory simulation experiments confirmed the observed field damages and revealed that top-load compression on the packages resulted in bruise damage in bananas while the simulated package drop resulted in severe neck injuries. Exposure to simulated vibration contributed to the development of fruit rub damage in the most top and bottom tier packages of the stacked column however, the middle tiers exhibited minor level of rubbing damage. Minimizing bruising and neck injuries in bananas requires careful storage and handling of packages especially during the distribution from the DC to retail stores. Further research is warranted to examine the failure mechanisms of paperboard packaging caused by moisture absorption during the ripening process as partial package failure was associated with increased neck injuries in bananas. Minimizing mechanical damage caused by vibration also requires further investigation on the vibration transmissibility and dampening properties of pallets and packaging.

\section{References}

1. S. R. Jaeger, L. Antúnez, G. Ares, J. W. Johnston, M. Hall, and F. R. Harker, "Consumers' visual attention to fruit defects and disorders: A case study with apple images," Postharvest Biology and Technology, vol. 116, pp. 36-44, 2016.

2. J. Ekman, N. King, S. Lindsay, and K. Gething, "Improved handling in banana supply chains," 2011.

3. J. Margetts, "Australian Banana Industry- Strategic Investment Plan," Plant \& Food Research Australia Pty Ltd 2014.

4. C. Day. (2018, 14/09/2018). Despite Higher Prices, Aussies Still Love their Bananas. Available: https://www.nielsen.com/au/en/insights/news/2018/ despite-higher-prices-aussies-still-love-theirbananas.html

5. B. K. Dadzie and J. E. Orchard, Routine postharvest screening of banana/plantain hybrids: criteria and methods: International Plant Genetic Resources Institute (IPGRI), 1997.

6. W. Wasala, D. Dharmasena, C. Dissanayake, and B. Tilakarathne, "Feasibility Study on Styrofoam Layer Cushioning for Banana Bulk Transport in a Local Distribution System," Journal of Biosystems Engineering, vol. 40, pp. 409-416, 2015.

7. J. S. Del Aguila, L. S. Heiffig-del Aguila, F. F. Sasaki, G. M. Tsumanuma, M. das Graças Ongarelli, M. H. F. Spoto, et al., "Postharvest modifications of mechanically injured bananas," Revista Iberoamericana de Tecnología Postcosecha, Hermosillo, vol. 10, pp. 73-85, 2010.

8. T. Kitchener, "Project BA13019 :Carton Management inthe Banana Industry," Kitchener Partners Pty Ltd16th December 2015.

9. L. C. B. Maia, V. M. Maia, L. C. C. Salomão, B. P. A. Salles, and R. F. Pegoraro, "Metabolic alterations in banana induced by mechanical damage," Unimontes Cientifica, vol. 17, pp. 27-34, 2015.

10. V. M. Maia, L. C. C. Salomão, D. L. Siqueira, R. Puschman, V. J. G. Mota Filho, and P. R. Cecon, "Physical and metabolic alterations in" Prata Anã" banana induced by mechanical damage at room temperature," Scientia Agricola, vol. 68, pp. 31-36, 2011. 
11. U. Chukwu, A. Olorunda, and R. Ferris, "Effect of Postharvest Injury on Weight Loss, Ripening Period, and Pulp Loss on Musa spp. Fruits," in Postharvest Technology and Commodity Marketing: Proceedings of a Postharvest Conference, 2 [9] Nov to 1 Dec 1995, Accra, Ghana, 1998, p. 132.

12. I. Fernando, J. Fei, R. Stanley, and H. Enshaei, "Measurement and evaluation of the effect of vibration on fruits in transit-Review," Packaging Technology and Science, pp. 1-16, 2018.

13. U. L. Opara and P. B. Pathare, "Bruise damage measurement and analysis of fresh horticultural produce-A review," Postharvest Biology and Technology, vol. 91, pp. 9-24, 2014.

14. Z. Li and C. Thomas, "Quantitative evaluation of mechanical damage to fresh fruits," Trends in Food Science \& Technology, vol. 35, pp. 138-150, 2014.

15. N. H. Banks and M. Joseph, "Factors affecting resistance of banana fruit to compression and impact bruising," Journal of the Science of Food and Agriculture, vol. 56, pp. 315-323, 1991.

16. N. H. Banks, C. A. Borton, and M. Joseph, "Compression bruising test for bananas," Journal of the Science of Food and Agriculture, vol. 56, pp. 223-226, 1991.

17. W. Wasala, D. Dharmasena, T. Dissanayake, and B. Thilakarathne, "Vibration simulation testing of banana bulk transport packaging systems," Tropical Agricultural Research, vol. 26, 2015.

18. P. More, D. Behere, S. Housalmal, S. Khodke, S. Jadhav, and A. Kakade, "Assessment of vibration damage to banana bunches simulated by vibration tester," International Journal of Tropical Agriculture, vol. 33, pp. 1419-1422, 2015.

19. N. F. Sommer, H. M. Laude, L. J. Berry, H. F. Madsen, S. C. Hoyt, J. N. Luthin, et al., "Surface discoloration of pears: Compression pads between fruit and carton lid effectively prevent transit bruising of jumble-packed Bartlett pears," Order, 1957.

20. D. Slaughter, R. Hinsch, and J. Thompson, "Assessment of vibration injury to Bartlett pears," Transactions of the ASAE, vol. 36, pp. 1043-1047, 1993.
21. Mohsenin, Physical Properties of Plant and Animal Materials: Structure, Physical Characteristics, and Mechanical Properties vol. 2: Gordon and Breach, 1986.

22. V. M. Maia, L. C. C. Salomão, D. L. Siqueira, R. Puschmann, V. J. G. Mota Filho, and P. R. Cecon, "Types and intensity of mechanical damages on'prata anã'bananas along the commercialization chain," Revista Brasileira de Fruticultura, vol. 30, pp. 365-370, 2008.

23. F. B. Da Costa, R. Puschmann, S. I. Moreira, J. I. R. Junior, and F. L. Finger, "Survey of mechanical injury in "Prata Ana'banana during shipping," Revista Verde de Agroecologia e Desenvolvimento Sustentável, vol. 5, pp. 72-78, 2010.

24. ASTM, "Packaging and Shipping Container Standards," West Conshohocken, PA: ASTM International, 2016.

25. K. Vursavus and F. Ozguven, "Determining the effects of vibration parameters and packaging method on mechanical damage in golden delicious apples," Turkish Journal of Agriculture and Forestry, vol. 28, pp. 311-320, 2004.

26. Y. Yuwana, "Impact Bruise Susceptabilities of Unripe Banana, Mango and Avacado," Indonesian Food Nutrition and Progress (IFNP), vol. Volume 4,, pp. 56-60, 1997.

27. B. Jarimopas, S. P. Singh, and W. Saengnil, "Measurement and analysis of truck transport vibration levels and damage to packaged tangerines during transit," Packaging Technology and Science, vol. 18, pp. 179-188, 2005.

28. D. Fischer, W. Craig, A. Watada, W. Douglas, and B. Ashby, "Simulated in-transit vibration damage to packaged fresh market grapes and strawberries," Applied Engineering in Agriculture, vol. 8, pp. 363366, 1992. 